\title{
QUALIDADE DE JUNTAS COLADAS COM LÂMINAS DE MADEIRA ORIUNDAS DE TRÊS REGIÕES DO TRONCO DE Eucalyptus grandis, Eucalyptus saligna E Pinus elliottii ${ }^{1}$
}

\author{
Benedito Rocha Vital ${ }^{2}$, Antônio da Silva Maciel $^{3}$ e Ricardo Marius Della Lucia ${ }^{2}$
}

\begin{abstract}
RESUMO - Este experimento teve como objetivo avaliar a resistência de juntas coladas formadas pelas combinações de lâminas provenientes de três posições no tronco da madeira de Eucalyptus grandis, Eucalyptus saligna e Pinus elliottii. Foram empregados adesivos à base de poliacetato de vinila de média e alta viscosidade e resorcinol-formaldeído nas gramaturas de $150 \mathrm{~g} / \mathrm{m}^{2}$, em face simples para o poliacetato de vinila de média e alta viscosidades e $300 \mathrm{~g} / \mathrm{m}^{2}$, em face dupla, para o adesivo resorcinólico. O teor médio de umidade das lâminas, no momento da colagem, foi igual a $14 \%$. Os valores médios mais elevados de resistência ao cisalhamento foram obtidos nas juntas produzidas com madeira de Eucalyptus saligna, coladas com adesivos de poliacetato de média viscosidade e resorcinol-formaldeído. A maior porcentagem de falha profunda na madeira foi obtida em juntas de madeira de Pinus elliottii, unidas com adesivo de poliacetato de alta viscosidade, seguidas das juntas de Eucalyptus grandis e coladas com adesivo de poliacetato de média viscosidade. As combinações de lâminas oriundas das seguintes posições no tronco: medula e casca, intermediária e casca e casca e casca resultaram em linhas de cola com maiores resistências ao cisalhamento.
\end{abstract}

Palavras-chave: Lâminas de madeira, adesivos, adesão, Eucalyptus grandis, Eucalyptus saligna, Pinus elliottii.

\section{QUALITY OF WOOD JOINTS GLUED WITH WOOD VENEERS FROM THREE TRUNK REGIONS OF Eucalyptus grandis, Eucalyptus saligna AND Pinus elliottii}

\begin{abstract}
The objective of this work was to evaluate the shear strength of glued wood joints from pith, outer and intermediary wood of Eucalyptus saligna, Eucalyptus grandis and Pinus elliottii. High and medium viscosity polyvinyl acetate and resorcinol-phenol adhesives were applied at spread rate of $150 \mathrm{~g} / \mathrm{m}^{2}$ in single line and at spread rate of $300 \mathrm{~g} / \mathrm{m}^{2}$ in double glue line for the resorcinolic adhesive. The mean wood moisture content was 14\%. Higher shear strength was obtained with Eucalyptus saligna veneer glued with medium viscosity polyvinyl resorcinolic adhesive. The highest percentage of wood failure was found on Pinus elliottii veneer glued with high viscosity polyvinyl acetate adhesive followed by Eucalyptus grandis glued with medium viscosity polyvinyl acetate. Veneer combinations with higher shear strength were pith/outer, intermediary/ outer and outer/outer wood.
\end{abstract}

Keywords: Wood veneer, adhesives, adhesion, Eucalyptus grandis, Eucalyptus saligna, and Pinus elliottii.

\footnotetext{
${ }^{1}$ Recebido em 21.01.2005 e aceito para publicação em 05.04.2006.

${ }^{2}$ Departamento de Engenharia Florestal da UFV, Viçosa-MG, Brasil. E-mail: <vital@ ufv.br>.

${ }^{4}$ Rua do Sampaio, 49, Grambery, 36010-360 Juiz de Fora-MG.
} 


\section{INTRODUÇÃO}

A adesão entre componentes de madeira, bem como entre outros materiais, depende de uma série de parâmetros relacionados às características físico-químicas do adesivo e do material a ser colado, do procedimento adotado na colagem, da forma geométrica e do tamanho das peças a serem coladas e, ainda, das condições a que as peças coladas serão expostas, quando em serviço (SOBRAL FILHO, 1982; MARRA, 1992). A resistência, a estabilidade e a vida útil de uma estrutura colada dependem, em grande parte, da resistência, rigidez e durabilidade das ligações.

No entanto, o emprego de adesivos pela indústria de base madeira, atualmente, é imprescindível. Segundo Marra (1980), mais de $70 \%$ dos produtos à base de madeira utilizam, de uma forma ou de outra, adesivos em sua confecção. Peças de dimensões limitadas, por exemplo, podem ser utilizadas na confecção de grandes elementos estruturais na forma de madeira laminada colada. A confecção de painéis de madeira sólida, de móveis, de madeiras compensadas e aglomeradas, que são produtos amplamente utilizados, depende do uso racional de adesivos.

Adesivos são substâncias que promovem a adesão entre dois substratos pela ação de forças atrativas intermoleculares de origem primária (eletrostáticas, covalentes ou metálicas) ou secundárias (Van der Walls) (PIZZI e MITTAL, 1994). Essas forças atrativas quando atuam adequadamente entre dois substratos podem promover uma resistência que pode superar as forças coesivas dos próprios substratos.

Existem várias teorias que procuram explicar o fenômeno da adesão, porém nenhuma delas é capaz de justificar todos os aspectos de aderência e, provavelmente, a combinação entre elas é que mais se aproxima da realidade. Com relação à madeira, duas das teorias mais importantes são o enganchamento o mecânico e a teoria da adsorção. Segundo a teoria do enganchamento mecânico, a penetração do adesivo num substrato poroso leva à formação de ganchos ou entrelaçamento mecânico do adesivo que se prende nas camadas superficiais da madeira após a cura e endurecimento do adesivo. A teoria da adsorção estabelece que a adesão é resultante do contato molecular entre dois materiais que desenvolvem forças de atração superficiais. Para que ocorra um contato íntimo e contínuo entre um adesivo e um substrato, é necessário que o adesivo umidifique a madeira, fenômeno que envolve a migração do adesivo para dentro da estrutura submicroscópica da parede celular expulsando ar, água e outros componentes estranhos à superfície de contato da madeira. O umedecimento depende da natureza molecular do adesivo, qualidade da superfície, mobilidade molecular do adesivo, pressão no adesivo e tempo disponível até que o endurecimento do adesivo a imobilize (GALEMBECK e GANDUR, 2001).

$\mathrm{Na}$ indústria madeireira, muitas pesquisas envolvendo adesivos foram dedicadas à otimização da adesão, em madeiras de uso já consagrado e em novas espécies de madeira com potencial para serem utilizadas na indústria moveleira e da construção civil, o que levou ao desenvolvimento, nas últimas décadas, de adesivos com características próprias para a colagem desses materiais. As madeiras de reflorestamento, principalmente às dos gêneros Eucalyptus e Pinus, têm-se mostrado bastante promissoras para atender à demanda dessas indústrias e têm sido bastante estudadas com o intuito de determinar as suas propriedades físico-mecânicas (OLIVEIRA, 1997; DELLA LUCIA e VITAL, 1980; NOGUEIRAe LAHR, 1992). Já se conhece o comportamento diante da colagem de várias espécies desse gênero (FAMER, 1972; DELLA LUCIA e VITAL, 1989; NASCIMENTO et al., 2001; SERPA, 2001; DELLALUCIAe VITAL, 1981). Algumas colam bem, outras produzem juntas, consideradas insatisfatórias.

Segundo Kollmann et al. (1975) e Marra (1992), algumas características intrínsecas da madeira promovem significativas interferências no processo de colagem. A variabilidade que ocorre na densidade e porosidade entre os lenhos inicial e tardio, cerne e alburno, os lenhos juvenil e adulto e, ainda, nas madeiras de reação, além da presença de extrativos nas cavidades dos elementos anatômicos, são os fatores que mais afetam o processo de adesão.

A densidade básica é considerada como um parâmetro da maior importância para a determinação da qualidade da madeira. No gênero Eucalyptus, a densidade básica pode variar entre espécies, entre árvores de uma mesma espécie e dentro de uma mesma árvore em função, principalmente, do ciclo de vida da árvore e das condições edafoclimáticas do povoamento. Vital et al. (1984) e Kollmann et al. (1975) relataram que, quanto mais velha é a árvore, maior é a sua densidade. Segundo esses autores, tal fato se deve ao espessamento das 
paredes celulares dos elementos estruturais. Carpim e Barrichelo (1984), estudando o comportamento de madeiras de eucalipto, relataram que a densidade básica pode variar de acordo com a posição na árvore, tanto no sentido radial quanto no longitudinal.

A densidade da madeira indica a quantidade aproximada de espaços vazios disponíveis para receber líquidos, por exemplo adesivos. Assim, quanto menor for a densidade, maior será a permeabilidade de sua estrutura (HUNT, 1962) e, provavelmente, mais forte será a ligação interfacial formada entre o adesivo e a madeira (MARRA, 1992 ). No entanto, quanto menor for a densidade da madeira, menor será a sua resistência mecânica (TSOUMIS, 1991) .

A estrutura dos anéis de crescimento também pode afetar a permeabilidade da madeira. Nas coníferas, o lenho inicial geralmente é mais permeável do que o lenho tardio, por apresentar células de maior diâmetro e paredes mais delgadas (AMARAL, 1967). Nas folhosas, a permeabilidade depende do número de poros por unidade de área, da sua distribuição e da ocorrência ou não de tiloses (TSOUMIS, 1991).

A presença de madeira juvenil por sua menor densidade, menor porcentagem de lenho tardio e elementos fibrosos de menor comprimento com paredes mais finas, com menor conteúdo de celulose, também pode interferir no processo de adesão. Além disso, a madeira juvenil é formada por anéis de crescimento mais largos, com menor resistência mecânica e contração e inchamento mais pronunciados no sentido longitudinal, em comparação com a madeira normal, e podem gerar tensões na linha de cola.

Essas variações podem afetar a mobilidade do adesivo e influenciar a sua cura. Geralmente, as madeiras de folhosas, devido à sua constituição química e maior complexidade estrutural, apresentam maior dificuldade de colagem que as coníferas.

Assim, este trabalho teve por objetivo avaliar a qualidade de juntas coladas, produzidas pela combinação de lâminas, provenientes de três posições no tronco da madeira de Eucalyptus grandis, Eucalyptus saligna e Pinus elliottii. A qualidade das juntas foi avaliada, determinando-se a sua resistência ao cisalhamento em tração e a porcentagem de falha profunda na madeira.

\section{MATERIAL E MÉTODOS}

Foram utilizadas, lâminas de madeira de Eucalyptus grandis, Eucalyptus saligna e Pinus elliottii, provenientes de árvores cultivadas em áreas do campus da Universidade Federal de Viçosa. As toras de árvores com idades de 50,40 e 40 anos, respectivamente, foram processadas na forma de tábuas com espessuras médias de $30 \mathrm{~mm}$ e secadas ao ar. Foram selecionadas tábuas diametrais que foram cortadas em seções de $600 \mathrm{~mm}$ de comprimento. As seções foram adicionalmente processadas, conforme mostrado na Figura 1, para se obterem amostras próximo da medula, próximo da casca e na região intermediária. De cada parte, depois de identificada, produziu-se um par de lâminas, que depois de usinadas mediam cerca de $600 \mathrm{~mm}$ de comprimento, $100 \mathrm{~mm}$ de largura e $5 \mathrm{~mm}$ de espessura. Para cada espécie foram produzidas lâminas suficientes para se obterem as seguintes combinações em função da posição no tronco: medula/medula; casca/ casca; intermediária/intermediária; intermediária/medula; intermediária/casca e medula/casca. Simultaneamente à obtenção das lâminas foram produzidos sarrafos dos quais se retiraram corpos-de-prova com dimensões aproximadas de 2,0 x 2,0 x 3,0 cm para a determinação da resistência ao cisalhamento por compressão da madeira sólida. Os pares de lâminas depois de identificados foram levados á estufa e deixados secar, à temperatura de $35^{\circ} \mathrm{C}$, até atingirem um teor médio de umidade de $14 \%$.

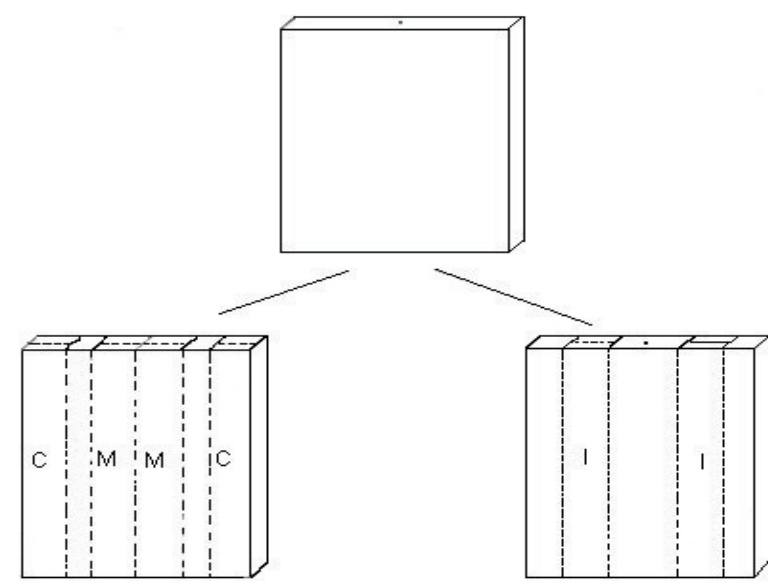

Figura 1 - Diagrama esquemático mostrando a obtenção dos pares de lâminas correspondentes a cada região da tábua, em que "C" se refere à região da casca, "M" à região da medula e "I" à região intermediária

Figure 1-Diagram showing the veneer pair production from each region, where " $C$ " refers to the outer region, " $M$ " to the pith region and " $I$ " to the intermediary region.

R. Árvore, Viçosa-MG, v.30, n.4, p.637-644, 2006 
Pouco antes de receber o adesivo, as superfícies foram lixadas, com lixa de grã 80, e devidamente limpas com a finalidade de apresentar as faces ativadas para a aplicação dos adesivos.

Os adesivos utilizados foram cedidos pela Alba Química, sendo empregados seguindo-se as recomendações especificadas nos respectivos Boletins Técnicos. Foram empregados adesivos em emulsão aquosa à base de poliacetato de vinila (PVAc) de média viscosidade (2000-4000 Cp) (Cascorez 2250) e alta viscosidade (4000-6000 Cp) Cascorez 2280 e resina à base de resorcinol-formaldeído (Cascophen-RS-216), com teores médios de sólidos iguais a 50,0; 51,3; e $55,5 \%$, respectivamente. Os adesivos em emulsão foram utilizados como recebidos, depois de homogeneizados, e a resina resorcinólica foi preparada pela mistura, em partes por peso, de: resina (100), catalisador (20). As quantidades aplicadas de cada adesivo foram iguais a $150 \mathrm{~g} / \mathrm{cm}^{2}$, em face simples para o poliacetato de vinila de média e alta viscosidade e $300 \mathrm{~g} / \mathrm{cm}^{2}$, em face dupla, para o resorcinol-formaldeído. Os adesivos foram preparados poucos minutos antes de seu emprego e espalhados sobre as superfícies com pincel. As juntas foram imediatamente fechadas e, assim, permaneceram por cerca de 15 minutos, sendo, então, prensadas a frio a $10 \mathrm{kgf} / \mathrm{cm}^{2}$ durante $8 \mathrm{~h}$. Foram confeccionados 162 pares de juntas, resultado de um arranjo fatorial completo do tipo $6 \times 3 \times 3 \times 3$, correspondentes às combinações entre posições, espécies de madeira, tipo de adesivo e três repetições.

Após a cura dos adesivos, as juntas foram deixadas em repouso, durante uma semana, em ambiente climatizado a $75 \pm 3 \%$ de umidade e $23 \pm 2{ }^{\circ} \mathrm{C}$ de temperatura, sendo depois desdobradas em 15 corpos de prova que foram devidamente identificados, voltando para as mesmas condições de climatização até atingir a umidade de equilíbrio higroscópico.

Foram selecionados, ao acaso, 10 corpos-de-prova para cada par de junta, cuja resistência ao cisalhamento em tração foi determinada seguindo-se a norma ASTM D2339-98 (1994). Após a ruptura, uma das metades do corpo-de-prova foi inspecionada com o auxílio de uma lâmina reticulada para quantificação da porcentagem de falha profunda na madeira.

Os resultados dos ensaios foram interpretados com o auxílio de análise de variância e do teste de Tukey a $5 \%$ de probabilidade.

R. Árvore, Viçosa-MG, v.30, n.4, p.637-644, 2006
Os ensaios de cisalhamento, em compressão, da madeira sólida foram executados seguindo os procedimentos da norma ASTM D143-83(1994), porém empregando-se amostras com dimensões nominais de $2 \times 2 \times 3 \mathrm{~cm}$. O teor médio de umidade da madeira no momento dos ensaios foi de $14 \%$. Após o rompimento dos corpos-de-prova foram selecionadas, ao acaso, 45 amostras da madeira de cada região para a determinação da densidade básica, utilizando-se o método de imersão em mercúrio, conforme descrito por Vital (1984).

\section{RESULTADOS E DISCUSSÃO}

No Quadro 1 estão apresentados os resultados médios de densidade básica e da resistência ao cisalhamento, em compressão, para madeiras oriundas das regiões da medula, casca e intermediária, além dos coeficientes de variação e dos números de amostras testadas.

O valor médio da densidade das madeiras em estudo assemelha-se àqueles encontrados na literatura (SERPA, 2001; PEREIRA et al., 2000). Nas madeiras de Eucalyptus, observou-se tendência de aumento no sentido medulacasca. Na madeira de Pinus, houve decréscimo inicial, na região próxima da medula e provavelmente contendo madeira juvenil. Posteriormente, houve acréscimo na direção da casca.

A resistência da madeira ao cisalhamento das madeiras oriundas da região próximo da casca foi sempre superior à resistência das madeiras oriundas da região próximo da medula.

A análise estatística indicou diferença significativa entre espécies, tipo de adesivo e combinações de lâminas. Observou-se, ainda, uma interação significativa entre espécie e tipo de adesivo.

No Quadro 2, mostram-se os valores médios de resistência ao cisalhamento das juntas coladas e porcentagem de falha profunda na madeira. Verificase que, entre as duas espécies de madeira de eucalipto, a que apresentou a maior média de resistência ao cisalhamento foi o Eucalyptus saligna, diferindo da média encontrada nas juntas coladas com Eucalyptus grandis e Pinus elliottii. Na porcentagem de falhas na madeira, os valores médios mais elevados foram observados nas juntas coladas com madeiras de Pinus elliottii e Eucalyptus grandis. Ao comparar os valores 
médios de resistência determinada nas juntas coladas com aqueles obtidos na madeira sólida, por exemplo na madeira localizada na posição intermediária, percebeuse que a relação entre esses valores foi aproximadamente de $64 \%$ para a madeira de Eucalyptus saligna, $56 \%$ para a madeira de Eucalyptus grandis e $48 \%$ para o Pinus elliottii.

De modo geral, esses valores foram inferiores aos esperados, uma vez que, segundo o Forest Products Laboratory (1999), a resistência da linha de cola ao cisalhamento paralelo à grã é superior à resistência da madeira para a maioria dos adesivos, com uma porcentagem de falha superior a $75 \%$. Apesar disso, a porcentagem de falhas encontrada neste experimento está compatível com aquelas constatadas por Nascimento (2002) para madeira de Eucalyptus e Pinus quando se empregou a mesma gramatura do adesivo resorcinólico.

$\mathrm{O}$ adesivo que proporcionou a maior média de resistência ao cisalhamento foi o poliacetato de viníla de média viscosidade, diferindo, estatisticamente, dos demais. Contudo, as maiores médias de porcentagem de falhas na madeira foram determinadas nas juntas coladas com adesivos de poliacetato de vinila de alta viscosidade.

Para a combinação de lâminas de madeira, a junta formada por madeira de medula/casca, intermediária/ intermediária e casca/casca foi a que apresentou os valores médios de resistência ao cisalhamento mais elevados, não havendo diferença estatística entre esses valores. Com relação à porcentagem de falha na madeira não houve diferença significativa em nenhuma combinação.

Quadro 1 - Valores médios de densidade $\left(\mathrm{g} / \mathrm{cm}^{3}\right)$ e resistência ao cisalhamento $\left(\mathrm{kgf} / \mathrm{cm}^{2}\right)$ das madeiras nas regiões da medula, casca e intermediaria

Table 1 - Mean density $\left(\mathrm{g} / \mathrm{cm}^{3}\right)$ and shear strength for pith, outer and intermediary wood

\begin{tabular}{|c|c|c|c|c|c|c|c|c|c|}
\hline \multirow[t]{4}{*}{ Características } & \multicolumn{9}{|c|}{ Espécies } \\
\hline & \multicolumn{3}{|c|}{ Eucalyptus grandis } & \multicolumn{3}{|c|}{ Eucalyptus saligna } & \multicolumn{3}{|c|}{ Pinus elliottii } \\
\hline & \multicolumn{3}{|c|}{ Posição no Tronco } & \multicolumn{3}{|c|}{ Posição no Tronco } & \multicolumn{3}{|c|}{ Posição no Tronco } \\
\hline & Medula & Casca & $\begin{array}{c}\text { Interme- } \\
\text { diária }\end{array}$ & Medula & Casca & $\begin{array}{c}\text { Interme- } \\
\text { diária }\end{array}$ & Medula & Casca & $\begin{array}{r}\text { Interme- } \\
\text { diária } \\
\end{array}$ \\
\hline Densidade $\left(\mathrm{g} / \mathrm{cm}^{3}\right)$ & 0,58 & 0,60 & 0,59 & 0,64 & 0,66 & 0,63 & 0,44 & 0,48 & 0,45 \\
\hline Núm. de amostras & & 45 & & & 45 & & & 45 & \\
\hline Coef. de variação (\%) & 12,0 & 9,5 & 11,1 & 11,0 & 13,0 & 11,6 & 10,0 & 11,0 & 12,0 \\
\hline $\begin{array}{l}\text { Resist. ao cisalhamento } \\
\left(\mathrm{kgf} / \mathrm{cm}^{2}\right)\end{array}$ & 131,0 & 158,1 & 153,4 & 116,9 & 164,3 & 155,6 & 95,1 & 118,5 & 115,9 \\
\hline Núm. de amostras & & 40 & & & 40 & & & 40 & \\
\hline Coef. de variação (\%) & 15,1 & 14,7 & 9,7 & 19,4 & 14,1 & 13,9 & 17,2 & 15,9 & 13,1 \\
\hline Teor médio de umidade $(\%)$ & & 14 & & & 14 & & & 14 & \\
\hline
\end{tabular}

Quadro 2 - Valores médios da resistência ao cisalhamento $\left(\mathrm{kgf} / \mathrm{cm}^{2}\right)$ e percentagem de falhas na madeira $(\%)$, segundo a espécie de madeira, tipo de adesivo e combinação de lâminas

Table 2 - Mean values for shear strength $\left(\mathrm{kgf} / \mathrm{cm}^{2}\right)$ and percentage of wood failure, according to wood species, adhesive and veneer combination

\begin{tabular}{|c|c|c|}
\hline Espécies de madeira & Tensão de ruptura $\left(\mathrm{Kgf} / \mathrm{cm}^{2}\right)$ & Falha na madeira $(\%)$ \\
\hline Eucalyptus saligna & $97,95 \quad \mathrm{~A}$ & $35,73 \quad \mathrm{C}$ \\
\hline Eucalyptus grandis & 86,14 B & 51,64 B \\
\hline Pinus elliottii & $55,63 \mathrm{C}$ & $65,12 \mathrm{~A}$ \\
\hline \multicolumn{3}{|l|}{ Tipo de adesivo } \\
\hline PVAc 2250 & $82,35 \quad \mathrm{~A}$ & $54,06 \quad \mathrm{~A}$ \\
\hline PVAc 2280 & $79,35 \quad \mathrm{~B}$ & 55,93 A \\
\hline Resorcinol & $77,72 \mathrm{C}$ & $42,49 \quad \mathrm{~B}$ \\
\hline \multicolumn{3}{|l|}{ Combinação de Lâminas } \\
\hline Medula/Casca & $83,02 \mathrm{~A}$ & $51,11 \mathrm{~A}$ \\
\hline Intermediária/Intermediária & 81,36 AB & $51,46 \mathrm{~A}$ \\
\hline Casca/Casca & $79,82 \mathrm{ABC}$ & $49,53 \mathrm{~A}$ \\
\hline Medula/Medula & $79,45 \quad \mathrm{BC}$ & $52,23 \mathrm{~A}$ \\
\hline Intermediária/Medula & 77,96 & $49,40 \mathrm{~A}$ \\
\hline Intermediária/Casca & 77,85 & $51,24 \mathrm{~A}$ \\
\hline
\end{tabular}

Em cada coluna, médias seguidas pela mesma letra não diferem estatisticamente, pelo teste de Tukey a 5\% de probabilidade. 
No Quadro 3, mostram-se os valores médios de resistência ao cisalhamento e a porcentagem de falha profunda na madeira, para o desdobramento da interação espécie de madeira e tipo de adesivo. Observa-se, nesse quadro, que o desdobramento e avaliação de espécies dentro do adesivo têm efeitos diferenciados entre espécies com relação a todos os adesivos testados, nas duas características avaliadas.O desdobramento e avaliação de adesivo dentro de espécie evidenciam que, para resistência ao cisalhamento, o adesivo PVAc2250 apresentou maior valor no Eucalyptus saligna e no Eucalyptus grandis. No Eucalyptus saligna, esse maior valor não diferiu daquele do adesivo resorcinólico, enquanto n o Eucalyptus grandis os valores foram diferentes entre os três adesivos testados. No Pinus elliottii, não se verificou diferença entre os adesivos com relação à resistência ao cisalhamento.

A porcentagem de falha na madeira, de Eucalyptus grandis apresentou o mesmo comportamento que a resistência ao cisalhamento. Em Eucalyptus saligna e Pinus elliottii, os adesivos apresentaram diferenças significativas entre si. Quanto a resistência ao cisalhamento, as médias mais elevadas foram obtidas nas juntas de Eucalyptus saligna, coladas com adesivos à base de poliacetato de média viscosidade e resorcinol- formaldeído. Nas juntas com madeira de Eucalyptus grandis, a maior resistência foi observada nas juntas coladas com adesivo de poliacetato de média viscosidade.

O comportamento diferenciado de cada tipo de adesivo dentro de cada espécie de madeira deve-se, possivelmente, à variabilidade na densidade e permeabilidade de cada tipo de madeira. Analisando os valores médios apresentados no Quadro 4, no que se refere à falha na madeira, nas juntas produzidas com madeira de Pinus e coladas com adesivo de poliacetato de vinila de alta viscosidade obtiveramse valores mais elevados. Nas madeiras de eucalipto, juntas produzidas com Eucalyptus grandis coladas com adesivo de poliacetato de média viscosidade apresentaram os maiores valores de falha na madeira.

Entre os adesivos, aquele à base de resorcinol produziu, em todas as espécies de madeira, menores porcentagens de falha. Tal fato pode estar associado ao tipo de filme formado pelo referido adesivo. Enquanto os adesivos de poliacetato de vinila formam um filme flexível e, portanto, mais suscetível à maior deformação, o adesivo resorcinólico forma um filme rígido e quebradiço, menos resistente às tensões aplicadas.

Quadro 3 - Valores médios de resistência ao cisalhamento $\left(\mathrm{kgf} / \mathrm{cm}^{2}\right)$ e porcentagem de falha na madeira $(\%)$, segundo a interação entre espécies de madeira e tipo de adesivo

Table 3 - Mean values for shear strength $\left(\mathrm{Kgf} / \mathrm{cm}^{2}\right)$ and percentage of wood failure, according to interaction between wood species and adhesive type

\begin{tabular}{|c|c|c|c|c|c|c|}
\hline \multirow[b]{2}{*}{ Tipo de Adesivo } & \multicolumn{3}{|c|}{ Tensão de Ruptura $\left(\mathrm{Kgf} / \mathrm{cm}^{2}\right)$} & \multicolumn{3}{|c|}{ Falha na Madeira $(\%)$} \\
\hline & $\begin{array}{c}\text { Eucalyptus } \\
\text { saligna }\end{array}$ & $\begin{array}{c}\text { Eucalyptus } \\
\text { grandis }\end{array}$ & $\begin{array}{c}\text { Pinus } \\
\text { elliottii }\end{array}$ & $\begin{array}{c}\text { Eucalyptus } \\
\text { saligna }\end{array}$ & $\begin{array}{c}\text { Eucalyptus } \\
\text { grandis }\end{array}$ & $\begin{array}{c}\text { Pinus } \\
\text { elliottii }\end{array}$ \\
\hline PVAc 2250 & $102,24 \quad$ A a & $91,27 \mathrm{~B} \mathrm{a}$ & $54,09 \mathrm{C} \mathrm{a}$ & $41,67 \mathrm{C} \mathrm{a}$ & $58,08 \mathrm{~B} \mathrm{a}$ & $\overline{68,06 \mathrm{~A} \mathrm{~b}}$ \\
\hline PVAc 2280 & $95,03 \mathrm{~A} \mathrm{~b}$ & $86,60 \mathrm{~B} \mathrm{~b}$ & $56,44 \mathrm{C}$ a & $35,69 \mathrm{C} \mathrm{b}$ & $52,44 \mathrm{~B} \mathrm{~b}$ & $74,06 \mathrm{~A} \mathrm{a}$ \\
\hline Resorcinol & 96,24 A a & $80,58 \mathrm{~B} \mathrm{c}$ & $56,36 \mathrm{C} \mathrm{a}$ & $29,73 \mathrm{C} \mathrm{c}$ & $41,42 \mathrm{~B} \mathrm{c}$ & $53,25 \mathrm{~A} \mathrm{c}$ \\
\hline
\end{tabular}

Para cada propriedade, médias seguidas pela mesma letra maiúscula ao longo da mesma linha e pela mesma letra minúscula ao longo da mesma coluna não diferem estatisticamente pelo teste de Tukey a 5\% de probabilidade.

\section{CONCLUSÕES}

As juntas coladas com madeira de Eucalyptus saligna tiveram os valores médios mais elevados de resistência ao cisalhamento e os menores valores de falha na madeira.

Os adesivos que proporcionaram os valores médios mais elevados de resistência da linha de cola ao cisalhamento e de porcentagem de falha na madeira foram o poliacetato de vinila de média e alta viscosidades, respectivamente.

As combinações medula/casca, intermediária/ intermediária e casca/casca, foram as que apresentaram as linhas de cola mais resistentes. Na combinação de lâminas, não houve efeito estatisticamente significativo para a porcentagem de falha profunda na madeira.

Considerando a interação entre as variáveis em estudo, os valores médios mais elevados de resistência ao cisalhamento foram determinados nas juntas 
produzidas com madeira de Eucalyptus saligna, coladas com adesivos de poliacetato de vinila de média viscosidade e resorcinol-formaldeído.

As maiores médias de porcentagem de falha profunda na madeira foram obtidas em juntas de madeira de Pinus elliottii, unidas com adesivo de poliacetato de vinila de alta viscosidade, seguidas das juntas de Eucalyptus grandis coladas com adesivo de poliacetato de média viscosidade.

De modo geral, tanto a resistência ao cisalhamento quanto a porcentagem de falha na madeira foi inferior ao esperado. Contudo, deve-se ressaltar que neste trabalho foi quantificada exclusivamente a porcentagem de falha profunda da madeira.

\section{REFERÊNCIAS BIBLIOGRÁFICAS}

\author{
AMARAL, C. M. A. Secagem de \\ madeiras. Contribuição para o \\ estudo da secagem das madeiras de \\ Pinus pinaster Sol., Quercus ilex L. e Populus \\ sp.. Lisboa: IS A, 1967.
}

\section{AMERICAN SOCIETY FOR TESTING AND} MATERIALS. - ASTM. Annual book of A.S.T.M. standards. Philadelphia: 1994. 478 p. v. 15.06 Adhesives.

\section{AMERICANSOCIETYFOR TESTINGAND}

MATERIALS. Annual book of A.S.T.M.

standards. Philadelphia: 1994.608 p. v. 04.10 Wood.

CARPIM, J.V.; BARRCHELO, L. E. G. Influência da procedência e clasee de diâmetro sobre as características da madeira de Eucalyptus grandis. In: CONGRESSO ANUAL DA ASSOCIAÇÃO BRASILEIRA DE CELULOSE E PAPEL, 17., São Paulo, 1984. Anais. São Paulo: ABCP, 1984. p.411-422

\section{DELLA LUCIA, R. M.; VITAL, B. R.}

Características físicas e mecânicas da madeira de Eucalyptys grandis W. Hill ex Maiden . Revista Árvore v. 4, n. 1, p. 70-74, 1980.

DELLA LUCIA, R. M.; VITAL, B. R. Ensaio de adesão das madeiras de Eucalyptus gummifera, E. paniculata e E. resinifera. Revista Árvore v. 13, n. 1, p. 98-106, 1989.
DELLA LUCIA, R. M.; VITAL, B. R. Avaliação da qualidade de juntas coladas de Madeira de 3 espécies de Eucalyptus. Revista Árvore, v. 5, n. 2, p. 172-180, 1981 .

FAMER, R. M. Handbook of

hardwoods. London: Her Majesty's Stationery Office, 1972. 244 p.

\section{FOREST PRODUCTS LABORATORY. Wood} handbook - Wood as na engineering material. Madison: U.S. Department of Agriculture, Forest Service, Forest Products Laboratory, 463 p. 1999. (Gen. Tech. Rep FPL-GTR-113).

GALEMBECK, F.; GANDUR, M. C. "Cientistas explicam o fenômeno da adesão". Química e Derivados. v. 393, p. 24-32. 2001.

HUNT,G. M.; GARRAT, G . A . Preservacion de la madera. Barcelona, Salvat, 1962.

KOLLMANN, F. F. P.; KUENZI, E.; STAM, A.J. Principles of wood science and technology. Berlin: Springer-Verlag, 1975. v. $2.703 \mathrm{p}$.

MARRA, G. G. Technology of wood bonding: principles in practice. New York: Van Nostrand Reinhold, 1992. 454 p.

MARRA, G. G. The role of adhesion and adhesives in products industry. In: WOOD ADHESIVES RESEARCH, APLLICATION AND NEEDS. 1980, Washington. Proceedings... Washington: USDA-PFL, 1980. p. 1-8.

MILLET, W. A.; GILLESPIE, R. H.; BAKER, R. J. Precision of rate-process method for predicting durability of adhesive bonds. In: ASTM. Durability of building materials and components, S.T.P.691. Philadelphia: 1980. p. 913-923.

NASCIMENTO, A. M.; DELLA LUCCIA, R. M.; VITAL, B. R. Colagem de emendas biseladas em madeiras de Pinus spp. e Eucalyptus citriodora. Revista Floresta e Ambiente, v. 8, p. 44-51, 2001.

R. Árvore, Viçosa-MG, v.30, n.4, p.637-644, 2006 
NASCIMENTO, A. M. et al. Comportamento de ligações adesivas em madeiras de reflorestamento. Floresta e Ambiente, v. 9, n. 1,p. 54-62, 2002.

NOGUEIRA, M. C. J. A .; LAHR, F. A. R. Indicações para o emprego de dezesseis espécies de Eucalyptus na construção civil. In: ENCONTRO BRASILEIRO EM MADEIRAS E EM ESTRUTURAS DE MADEIRA, 4.,1992, São Carlos. Anais... São Carlos : LaMEM-EESC-USP,1992. v. 1, p. 37-48.

OLIVEIRA, J. T. Caracterização da madeira de eucalipto para a construção civil. 1997. 429f. Tese (Doutorado em Engenharia Civil) - Universidade de São Carlos, São Carlos, 1997.

PEREIRA, J. C. D. et al. Características da madeira de algumas espécies de eucalipto plantadas no Brasil. Colombo: EMBRAPA Florestas, 2000.113 p. (EMBRAPA/MAA, Documentos, 38)

PIZZI, A.; MITTAL, K. L. Handbook of adhesive technology. New York: Marcell Deckker, 1994. 680 p.

R. Árvore, Viçosa-MG, v.30, n.4, p.637-644, 2006
SERPA, P. N. Avaliação da madeira de Eucalyptus grandis W. Hill ex Maiden, Eucalyptus saligna Smith e Pinus elliottii Engelm. para a produção de móveis. 2001.82f. Dissertação (Mestrado em Ciência Florestal) - Universidade Federal de Viçosa, Viçosa, 2001.

SOBRAL FILHO, M. Adesão e adesivos para madeira: teorias de adesão e a natureza das colagens com madeira. Brasília: Ministério da Agricultura, 1982. 22 p. (Série Técnica, 5).

TSOUMIS, G. Science and Technology of wood: structure, properties, utilization. New York: Van Nostrand Reinhold, 1991. 494 p.

VITAL, B. R. Métodos de determinação da densidade da Madeira. Viçosa, MG: SIF, 1984. 21 p. (SIF, Boletim Técnico,1)

VITAL, B . R. et al. Efeito da idade da árvore na densidade da madeira de Eucalyptus grandis cultivada na região do cerrado de Minas Gerais. Brasília: IBDF,1984. p. 41-52. 